\title{
Casimir interaction between a dielectric nanosphere and a metallic plane
}

\author{
Antoine Canaguier-Durand, ${ }^{1}$ Antoine Gérardin, ${ }^{1}$ Romain Guérout, ${ }^{1}$ Paulo A. Maia Neto, ${ }^{2}$ \\ Valery V. Nesvizhevsky, ${ }^{3}$ Alexei Yu. Voronin, ${ }^{4}$ Astrid Lambrecht, ${ }^{1}$ and Serge Reynaud ${ }^{1}$ \\ ${ }^{1}$ Laboratoire Kastler Brossel, CNRS, ENS, UPMC, case 74, 75252 Paris, France \\ ${ }^{2}$ Instituto de Física, UFRJ, CP 68528, Rio de Janeiro, RJ, 21941-909, Brazil \\ ${ }^{3}$ Institut Laue-Langevin, 6 rue Jules Horowitz, BP 156, 38042 Grenoble, France \\ ${ }^{4}$ P.N. Lebedev Physical Institute, 53 Leninsky prospect, 117924 Moscow, Russia
}

(Dated: November 6, 2018)

\begin{abstract}
We study the Casimir interaction between a dielectric nanosphere and a metallic plane, using the multiple scattering theory. Exact results are obtained with the dielectric described by a Sellmeier model and the metal by a Drude model. Asymptotic forms are discussed for small spheres, large or small distances. The well-known Casimir-Polder formula is recovered at the limit of vanishingly small spheres, while an expression better behaved at small distances is found for any finite value of the radius. The exact results are of particular interest for the study of quantum states of nanospheres in the vicinity of surfaces.
\end{abstract}

PACS numbers:

\section{INTRODUCTION}

The Casimir effect, due to the scattering of quantum fluctuations of the electromagnetic vacuum [1], is the dominant interaction between neutral bodies at distances large compared to atomic scales [2, 3] (more references in [4]). For this reason, it has a strong impact in various important domains, such as atomic and molecular physics, condensed matter and surface physics, chemical and biological physics, micro- and nano-technology [5].

In this paper, we will consider the case of dielectric nanospheres in the vicinity of a metallic surface. This study is part of a discussion of the intriguing phenomenon of small heating of ultra-cold neutrons (UCN) in traps [68] which could be explained by the interaction between UCN and nanospheres levitated in the quantum states created by the interaction of nanospheres with surfaces [9]. In order to characterize this phenomenon and compute the properties of the quantum states, one needs to have a detailed and careful treatment of the interaction potential. In particular, as shown below, the commonly used Casimir-Polder formula [10] is not sufficient to this purpose.

Below we first recall how the scattering formula [1] can be applied to the study of a sphere of radius $R$ at a distance $L$ of closest approach to the plane. We then give numerical evaluations and graphical plots of the interaction energy. The Casimir-Polder formula is recovered when the radius of the nanosphere is smaller than all other length scales. The short and long distance limits are then found to show subtle interplays with the limit of small radius.

Important consequences of these results are obtained for the behavior of the interaction at small distances. While the Casimir-Polder energy scales as $L^{-3}$ at small values of $L$, the full expression is found to be better behaved for any finite value of $R$, which leads to a regular solution for the quantum states.

\section{SCATTERING FORMALISM}

In this paper, we will not consider the effect of thermal fluctuations on the interaction (they are expected to be small at the not so large separations considered here). We thus start from the scattering formula for the Casimir energy at zero temperature [11]

$$
\begin{array}{ll}
E=\hbar \int_{0}^{\infty} \frac{\mathrm{d} \xi}{2 \pi} \ln \operatorname{det} \mathcal{D}, & \mathcal{D}=(I-\mathcal{M}) \\
\mathcal{M}=\mathcal{R}_{S} e^{-\mathcal{K} \mathcal{L}_{\mathcal{R}}} e^{-\mathcal{K} \mathcal{L}}, & \mathcal{L}=L+R
\end{array}
$$

The Casimir energy is written in terms of reflection operators $\mathcal{R}_{S}$ and $\mathcal{R}_{P}$ which describe the diffraction by the sphere and the plate. These operators are evaluated with reference points at the sphere center and at its projection on the plane, respectively. The operator $e^{-\mathcal{K} \mathcal{L}}$ accounts for one-way propagation along the distance $\mathcal{L}=L+R$ separating these two points. The operator $\mathcal{M}$ thus represents one round-trip propagation inside the cavity formed by the two surfaces. All quantities are written at imaginary frequencies $\omega=i \xi$ after a Wick rotation.

The scattering formula (1) provides a compact way of taking the multiple scatterings between the interacting bodies into account. It can be considered as generalizing the Dzyaloshinskii-Lifshitz-Pitaevskii formula [12] to arbitrary scattering properties of the two bodies. It can be applied in various geometries and has in particular been recently used for calculating the Casimir interaction between a metallic sphere and a metallic plane [13 16] (see also [17, 18]). In the following, we use the same techniques and notations as in [15, 16] and apply them to the case of a dielectric nanosphere above a metallic plane.

The reflection on the plane is conveniently written by using a plane-wave basis $|\mathbf{k}, \pm, p\rangle$ where $\mathbf{k}$ is the wavevector component parallel to the plane $x y$ of the metallic surface, $p=$ TE, TM the polarization, + $/-$ the upwards/downwards propagation direction. This basis is well adapted to the description of the propagation op- 
erator $e^{-\mathcal{K} \mathcal{L}}$, since $\mathcal{K}$ is thus diagonal with elements $\kappa=\sqrt{\xi^{2} / c^{2}+k^{2}}$ representing the (Wick rotated) wavevector $z$-component for the imaginary frequency $\xi$. The reflection operator $\mathcal{R}_{P}$ preserves all plane wave quantum numbers but the propagation direction, and its elements are given by the standard Fresnel specular reflection amplitudes for an homogenous medium.

Then the reflection on the sphere is more easily written by using the multipole basis $|\ell m P\rangle$, with $\ell(\ell+1)$ and $m$ denoting the angular momentum eigenvalues (with $\ell=1,2, \ldots, m=-\ell, \ldots, \ell)$ and $P=E, M$ representing electric and magnetic multipoles. The reflection operator $\mathcal{R}_{S}$ has its elements given by the standard Mie scattering amplitudes. Thanks to rotational symmetry, the operator $\mathcal{M}$ commutes with the angular momentum operator $J_{z}$. Hence $\mathcal{M}$ is block diagonal, and each block $\mathcal{M}^{(m)}$ (corresponding to a given subspace $m$ ) yields an independent contribution to the Casimir energy

$$
\begin{aligned}
& E=\frac{\hbar}{\pi} \int_{0}^{\infty} \mathrm{d} \xi \sum_{m}^{\prime} \ln \operatorname{det}\left(I-\mathcal{M}^{(m)}\right) \\
& \mathcal{M}^{(m)}=\left(\begin{array}{ll}
\mathcal{M}_{E E}^{(m)} & \mathcal{M}_{E M}^{(m)} \\
\mathcal{M}_{M E}^{(m)} & \mathcal{M}_{M M}^{(m)}
\end{array}\right)
\end{aligned}
$$

The primed sum is a sum over positive integers with the term $m=0$ counted for its half. We have organized $\mathcal{M}^{(m)}$ in terms of block-matrices built up on electric and magnetic contributions. The corresponding matrix elements are given and discussed in [16].

We now apply these formulas to the case of a dielectric nanosphere above a metallic plane. In particular, the plots shown below will be calculated for the case of interest for UCN studies [9], namely a diamond nanosphere above a copper plane. We model copper dielectric response with a Drude model

$$
\varepsilon(i \xi)=1+\frac{\omega_{P}^{2}}{\xi(\xi+\gamma)}
$$

This is written at imaginary frequencies $\omega=i \xi$, with $\omega_{P}^{2}$ the squared plasma frequency proportional to the density of conduction electrons in the metal, and $\gamma$ the damping rate which measures the relaxation of these electrons. For explicit calculations and plots, we will use the relations $\omega_{P}=2 \pi c / \lambda_{P}$ with the plasma wavelength $\lambda_{P}=136 \mathrm{~nm}$ and $\gamma=0.0033 \omega_{P}$. As $\gamma$ is small when compared to $\omega_{P}$ for a good metal such as copper, its influence is small at the not too large distances considered in the present study (see [19] for more details and references). The diamond dielectric response is described by a Sellmeier model

$$
\varepsilon(i \xi)=1+\sum_{i} \frac{B_{i} \omega_{i}^{2}}{\omega_{i}^{2}+\xi^{2}}
$$

For diamond, a good enough description will be obtained with a single component in this formula with $B_{1}=4.91$ and $\omega_{1}=2 \pi c / \lambda_{1}$ with the wavelength $\lambda_{1}=106 \mathrm{~nm}$. The

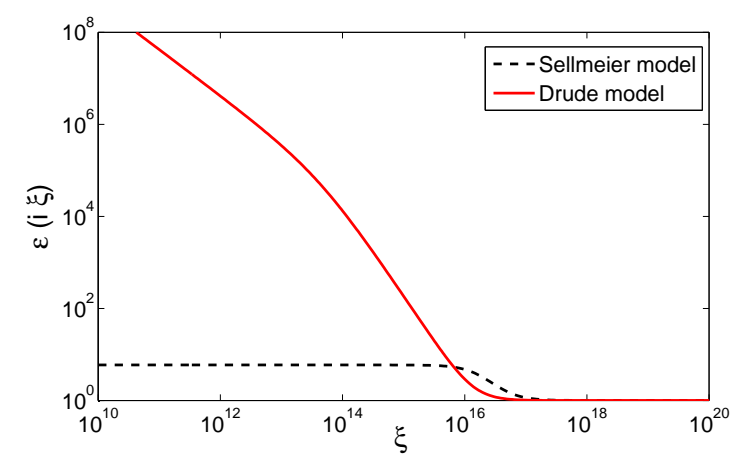

FIG. 1: Relative permittivity $\varepsilon(i \xi)$ for copper (Drude model; red line) and diamond (Sellmeier model; black dashed).

damping is disregarded here since it do not play any significant role.

The two permittivities are shown on Fig 1 where the black dashed curve is for diamond, and the plain red curve for copper. For copper, $\varepsilon(i \xi)$ is very large for $\xi$ much smaller than $\omega_{P}$, which means that the metal tends to become a very good reflector. For diamond, $\varepsilon(i \xi)$ tends to its static value $\varepsilon(0)=1+B_{1}$ for $\xi$ much smaller than $\omega_{1}$. For larger frequencies in contrast, the dielectric properties become poorer for diamond as well as copper. As $\omega_{P}$ and $\omega_{1}$ have similar values, we thus expect a transition to take place between van der Waals and Casimir-Polder regimes [10] when the distance between two objects is of the order of $\lambda_{P}$ or $\lambda_{1}$. The simple models (3.4) are sufficient for the purpose of the present work. They could easily be improved to take into account interband transitions for copper, multiple components and damping in the Sellmeier model for diamond.

\section{NUMERICAL EVALUATIONS}

For evaluating the determinant in (1] 2), one needs to truncate the vector space at some maximum value $\ell_{\max }$ of angular momentum [13 16]. A qualitative understanding of the associated effects may be obtained from the localization principle [20]: the value of $\ell_{\max }$ required for a given accuracy level is expected to scale with the size parameter $\hat{\xi} R$ (where $\hat{\xi}=\xi / c$ ) which captures the dependence of scattering amplitudes on the sphere radius. Meanwhile, the frequencies giving the main contribution to the Casimir energy scale as $\hat{\xi} \sim 1 / L$. As a consequence, the required $\ell_{\max }$ scales as $R / L$ for intermediate and short separation distances. In the present paper, we will be interested in nanospheres $R \leq 20 \mathrm{~nm}$ so that calculating with $\ell_{\max }=100$ will be sufficient for a good accuracy for the results discussed below.

The numerical results are shown in Fig 2 for different values of $R(2,5,10$, and $20 \mathrm{~nm})$. We plot the absolute value $|E|$ of the Casimir energy $(E \leq 0)$ with respect to the distance $L$, from 1 to $500 \mathrm{~nm}$. We see 


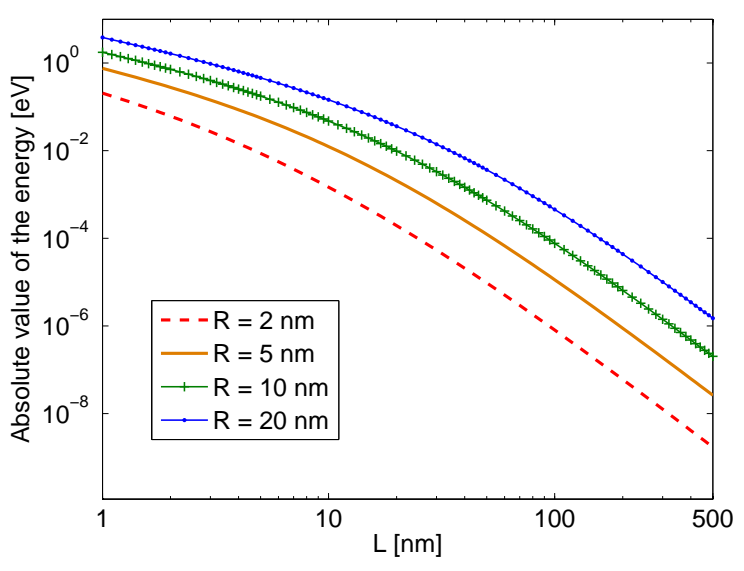

FIG. 2: Absolute value $|E|$ of the Casimir energy $E$ (measured in $\mathrm{eV}$ ) with respect to the distance $L$ for nanospheres of radii $R=2,5,10,20 \mathrm{~nm}$. Logarithmic scales are used on both axis.

that two regimes appear, which are reminiscent of non retarded van der Waals regime at short distances [21], and retarded Casimir-Polder regime at large distances [10], with different dependences upon the distance $L$ (see the slopes of the curves) and the radius $R$ (see the vertical spacing between the curves). Our results do not coincide with these well known limits because they take into account higher order multipole contributions to the scattering upon the sphere 22]. Of course, the CasimirPolder expression will be recovered for small values of the radius, as shown in the next section.

\section{LIMIT OF SMALL NANOSPHERES}

In this section, we consider the limit of a punctual sphere, when the radius $R$ is smaller than any other length scale (in loose terms, we take the limit $R \rightarrow 0$ ). We show that the Casimir-Polder expressions are recovered, as expected. As the derivations presented here are applied to dielectric nanospheres, we will use the fact that the permittivity for the nanosphere remains finite at all frequencies. We also keep the same description of the dielectric response for the small values of the radius considered here. Note that for metallic nanospheres in contrast, it would be necessary to take into account the confinement effect for conduction electrons [23, 24].

We start from the formulas (1-2) giving the Casimir energy for a sphere of radius $R$ at a distance $L$ of closest approach to a plane. Reflection on the plane is described by Fresnel amplitudes $r_{\mathrm{TE}}$ and $r_{\mathrm{TM}}$ while scattering on the sphere is described by Mie amplitudes $a_{\ell}$ and $b_{\ell}$ (defined as in [25]). The following expressions, valid at low values of the parameter $R \hat{\xi}$, are sufficient for calculating the energy at the limit $R \rightarrow 0$

$$
\begin{aligned}
& a_{\ell} \simeq(-1)^{\ell} \frac{\ell+1}{\ell \varepsilon+\ell+1} \frac{(\varepsilon-1)(R \hat{\xi})^{2 \ell+1}}{(2 \ell+1) ! !(2 \ell-1) ! !} \\
& b_{\ell} \simeq(-1)^{(\ell+1)} \frac{(\varepsilon-1)(R \hat{\xi})^{2 \ell+3}}{(2 \ell+3) ! !(2 \ell+1) ! !}
\end{aligned}
$$

As the dimensionless number $R \hat{\xi}$ is much smaller than unity (for $L \hat{\xi} \sim 1$; see the discussion in the previous section) and $\varepsilon$ remains finite at all frequencies, it follows that all these amplitudes are small and, simultaneously that the amplitude $a_{1}$ dominates all other Mie amplitudes.

The calculation of the energy is therefore much simpler than in the general case. As a first simplification, one may indeed replace the non linear expression (2) by a linearized one (perturbative approximation)

$$
E=-\frac{\hbar}{\pi} \int_{0}^{\infty} \mathrm{d} \xi \sum_{m}^{\prime} \operatorname{tr} \mathcal{M}^{(m)}
$$

Then, one may keep only the contributions to this sum which are proportional to the amplitude $a_{1}$ (electric dipolar approximation)

$$
\begin{aligned}
& E_{1} \simeq-\frac{\hbar}{\pi} \int_{0}^{\infty} \mathrm{d} \xi\left(\frac{1}{2} \mathcal{M}_{E E}^{(0)}+\mathcal{M}_{E E}^{(1)}\right) \\
& \mathcal{M}_{E E}^{(0)}=-\frac{3}{2} \frac{a_{1}}{\hat{\xi}^{3}} \int_{0}^{\infty} \frac{k^{3} \mathrm{~d} k}{\kappa} r_{\mathrm{TM}} e^{-2 \kappa L} \\
& \mathcal{M}_{E E}^{(1)}=\frac{3}{4} \frac{a_{1}}{\hat{\xi}^{3}} \int_{0}^{\infty} \frac{k \mathrm{~d} k}{\kappa}\left(\hat{\xi}^{2} r_{\mathrm{TE}}-\kappa^{2} r_{\mathrm{TM}}\right) e^{-2 \kappa L}
\end{aligned}
$$

We also rewrite $a_{1}$ in terms of a dynamical electric polarizability $\alpha(\xi)$ defined for the small nanosphere $\left(\alpha R^{3}\right.$ is a reduced polarizability having the dimension of a volume; the SI polarizability is $\varepsilon_{0} \alpha R^{3}$ )

$$
a_{1}=-\frac{2}{3} \alpha R^{3} \hat{\xi}^{3} \quad, \quad \alpha=\frac{\varepsilon-1}{\varepsilon+2}
$$

Collecting the results, we finally recover the full CasimirPolder formula [10] as written in [26, 27]

$$
\begin{aligned}
& E_{1} \simeq-\frac{\hbar c R^{3}}{2 \pi} \int_{0}^{\infty} \mathrm{d} \hat{\xi} \alpha(\hat{\xi}) \\
& \times \int_{0}^{\infty} \frac{k \mathrm{~d} k}{\kappa}\left(\hat{\xi}^{2}\left|r_{\mathrm{TE}}\right|+\left(k^{2}+\kappa^{2}\right)\left|r_{\mathrm{TM}}\right|\right) e^{-2 \kappa L}
\end{aligned}
$$

We have used the fact that $r_{\mathrm{TE}}(i \xi)<0$ and $r_{\mathrm{TM}}(i \xi)>0$.

We repeat at this point that the formula (9) has been obtained after two simplifications corresponding to the perturbative approximation and electric dipolar approximation. As the Casimir-Polder interaction between atoms [10], it contains in particular the limits of non retarded van der Waals and retarded Casimir-Polder expressions [26, 27]. The energy scales in both cases as $R^{3}$ that is also the volume of the sphere. This result means 
that the nanosphere behaves at the limit $R \rightarrow 0$ as a big atom with an electric polarizability $\alpha R^{3}$. As we will see in the next section, this simple behavior does not remain true for arbitrary values of the radius $R$.

\section{ASYMPTOTIC BEHAVIOR AT SHORT AND LONG DISTANCES}

In this section, we discuss the asymptotic behaviors of the Casimir energy $E$ at short and long distances.

As a first step to this aim, we write a Casimir-Polder formula $E_{\mathrm{CP}}$ deduced from $E_{1}$ in the limit $L \gg \lambda_{P}, \lambda_{1}$ where copper may be considered as perfectly reflecting and diamond as having a constant electric polarizability

$$
\begin{aligned}
& E_{\mathrm{CP}}=-\frac{4 \pi c_{4} R^{3}}{3 L^{4}} \\
& c_{4}=\frac{9 \hbar c \alpha_{0}}{32 \pi^{2}}, \quad \alpha_{0}=\frac{\varepsilon(0)-1}{\varepsilon(0)+2}
\end{aligned}
$$

We proceed similarly with the van der Waals prediction $E_{\mathrm{vdW}}$ deduced from $E_{1}$ for $L \ll \lambda_{P}, \lambda_{1}$

$$
\begin{aligned}
& E_{\mathrm{vdW}}=-\frac{4 \pi c_{3} R^{3}}{3 L^{3}} \\
& c_{3}=\frac{3 \hbar c \alpha_{0}}{16\left(\sqrt{2} \lambda_{P}+\sqrt{1-\alpha_{0}} \lambda_{1}\right)}
\end{aligned}
$$

The values $E_{\mathrm{CP}}$ and $E_{\mathrm{vdW}}$ are equal at a crossing length

$$
L_{*}=\frac{c_{4}}{c_{3}}=\frac{3\left(\sqrt{2} \lambda_{P}+\sqrt{1-\alpha_{0}} \lambda_{1}\right)}{2 \pi^{2}}
$$

that is approximately $39 \mathrm{~nm}$ with the values corresponding to copper and diamond.

As expected from already presented qualitative arguments, the exact Casimir expression $E$ (given by eq 10 is well approximated by $E_{\mathrm{CP}}$ when $R$ is the smallest length scale and $L$ the largest one

$$
E \simeq E_{\mathrm{CP}}, \quad R \ll L_{*} \ll L
$$

Meanwhile, the exact Casimir expression $E$ is well approximated by $E_{\mathrm{vdW}}$ when $R$ is the smallest length scale and $L$ smaller than $L_{*}$

$$
E \simeq E_{\mathrm{vdW}}, \quad R \ll L \ll L_{*}
$$

Of course, there exist a variety of behaviors when the two latter conditions are not met.

In order to explore this variety, we plot on Fig 3 the logarithmic slope (log-log derivative) of the energy $|E|$ versus distance $L$

$$
\nu=-\frac{\partial \ln |E|}{\partial \ln L}=\frac{L F(L)}{E(L)} \quad, \quad F(L)=-\frac{\partial E}{\partial L}
$$

The parameter $\nu$ would be a constant if the energy $|E|$ obeyed a power law dependence $1 / L^{\nu}$ (for example $\nu=4$

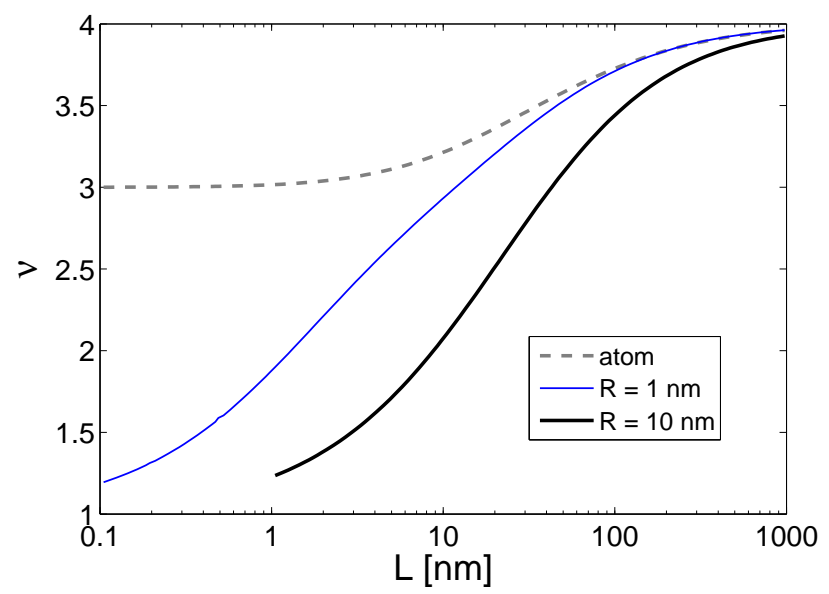

FIG. 3: Logarithmic slope $\nu$ as a function of the distance $L$. Plain curves represent the nanosphere case with different values for the radius $R$, dashed curve represents the atomic limit, that is a nanosphere with $R \rightarrow 0$.

for $E_{\mathrm{CP}}$ or $\nu=3$ for $\left.E_{\mathrm{vdW}}\right)$. In the general case, $\nu$ depends on $L$ and can thus be understood as describing a 'local' power law in the vicinity of $L$.

We see on Fig. 3 that $\nu$ tends to the expected value 4 at large distances. At small distances in contrast, the van der Waals value $\nu=3$ is never a good approximation, which can be understood by inspecting the conditions for (14) to be true. For any finite value of the radius $R$, we have indeed to cross the conditions $L \sim R$ when the distance is decreased and (14) can no longer be valid after this crossing.

Another facet of the same problem becomes apparent when looking at the dependence of $E$ versus $R$. We plot on Fig 4 a logarithmic slope $\mu$ calculated as in (15)

$$
\mu=\frac{\partial \ln |E|}{\partial \ln R}
$$

The parameter $\mu$ would be a constant if $|E|$ obeyed a simple power law dependence $R^{\mu}$. In particular the volumetric value $\mu=3$ is obtained for both $E_{\mathrm{CP}}$ and $E_{\mathrm{vdW}}$. We see on Fig. 4 that $\mu$ approaches this value at the limit of large distances, but departs from it everywhere else, anew indicating that $E_{\mathrm{vdW}}$ is not a good approximation.

We now give an improved version of the van der Waals formula (11) which explains some of the features of the exact energy $E$. As $E_{\mathrm{vdW}}$ has been demonstrated above in the limit of a punctual sphere $R \rightarrow 0$, we may improve it for a finite size of the sphere through a pairwise summation over the volume. We thus obtain the Hamaker expression 28]

$$
\begin{aligned}
\overline{E_{\mathrm{vdW}}} & \simeq-\pi c_{3}\left(\frac{2 R(L+R)}{L(L+2 R)}-\ln \frac{L+2 R}{L}\right) \\
\overline{E_{\mathrm{CP}}} & \simeq-\frac{4 \pi c_{4} R^{3}}{3 L^{2}(L+2 R)^{2}}
\end{aligned}
$$




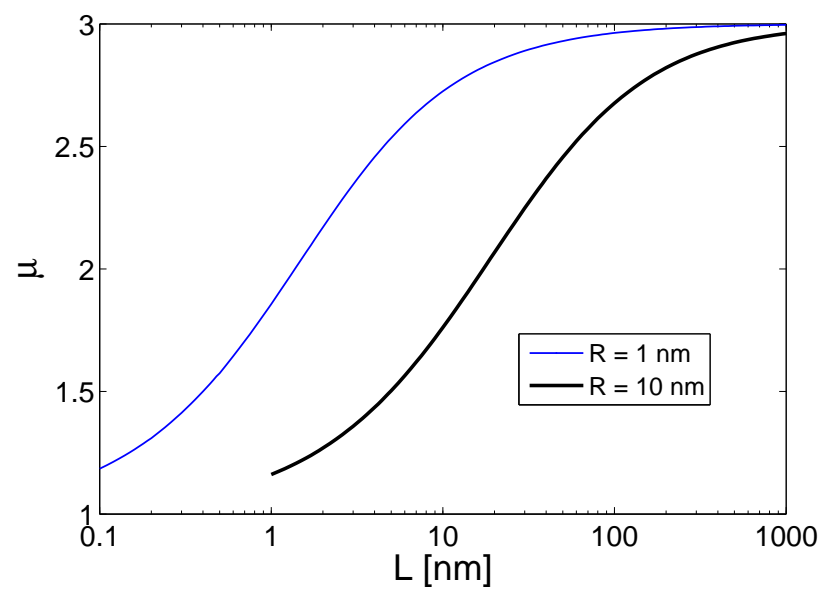

FIG. 4: Logarithmic slope $\mu$ for variation with the radius $R$, as a function of the distance $L$. Plain curves represent the nanosphere case with different values for the radius $R$, the atom case being in comparison a constant $\mu=3$.

For completeness, we did proceed similarly with the Casimir-Polder formula $E_{\mathrm{CP}}$.

These results allow one to understand the behaviors apparent on Figs 3.4. Let us again consider that we start from small nanospheres $R \ll L_{*}$ at large distances $L \gg$ $L_{*}$. Using the expression $E_{\mathrm{CP}}$, one obtains $\nu=4$ and $\mu=3$. When the distance $L$ is decreased, we cross two transitions $L \sim L_{*}$ and $L \sim R$ and end up with the formula $\overline{E_{\mathrm{vdW}}}$ for which we get $\nu=1$ and $\mu=1$. This line of reasoning reproduces the global variations seen on Figs 3,4 ,

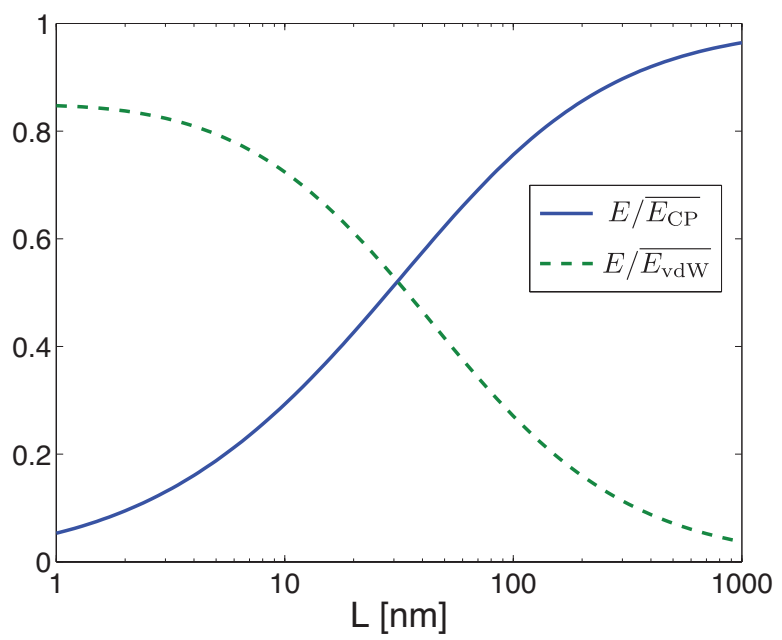

FIG. 5: Ratios of the exact energy $E$ to the expressions $\overline{E_{\mathrm{CP}}}$ (plain blue curve) and $\overline{E_{\mathrm{vdW}}}$ (dashed green curve). The two curves are calculated for $R=10 \mathrm{~nm}$.

In order to assess the quality of the estimations (17), we now plot on Fig 5 the ratios $E / \overline{E_{\mathrm{CP}}}$ and $E / \overline{E_{\mathrm{vdW}}}$. As expected, we find that $\overline{E_{\mathrm{CP}}}$ tends to reproduce the result
$E$ of the full numerical computation at large distances. We also see that $\overline{E_{\mathrm{vdW}}}$ obeys the same power law than $E$ at small distances (ratio tending to a constant value), but fails to predict the correct magnitude (the limit of the ratio is not 1). This feature can be understood through a close inspection of the case where $L$ is the smallest of all length scales.

When $L \ll R$, we can use the proximity force approximation and express the plane-sphere result in terms of the plane-plane one 29]. We are thus left with the evaluation of the Casimir effect between copper and diamond plane plates. As $L \ll L_{*}$, it is then possible to use the method designed in [30] to find

$$
E_{\mathrm{PFA}} \simeq-\pi c_{3}^{\prime} \frac{R}{L}, \quad L \ll R, L_{*}
$$

This expression shows the same functional dependencies than the Hamaker expression $\overline{E_{\mathrm{vdW}}} \sim-\pi c_{3} R / L$ while giving a different proportionality constant. This difference is due to the fact that (17) has been obtained through a pairwise integration of van der Waals forces whereas (18) has been calculated by taking into account the multiple interferences occuring in the FabryPerot cavity [30]. The numerical values thus obtained $\left(c_{3}^{\prime}=0.84 c_{3}\right.$ with the numbers corresponding to diamond and copper) explain the behavior seen on Fig 5 .

\section{CONCLUSIONS}

In this paper, we have presented an exact calculation of the Casimir interaction between a dielectric nanosphere and a metallic plane, using the multiple scattering formalism as developed recently for the plane-sphere geometry 13 16]. In order to discuss qualitatively the results obtained in this manner, we have also investigated the limits of a punctual sphere as well as the asymptotic behaviors at short and long distances.

This study has important applications for discussing the intriguing phenomenon of heating of ultra-cold neutrons in traps [6 -8]. This heating could be explained by the interaction between UCN and nanospheres levitated in the quantum states created by their interaction with surfaces [9]. In order to characterize quantitatively this phenomenon, a detailed knowledge of the interaction potential is required. In particular, the small-distance behavior of the Casimir energy plays a crucial role in the determination of the quantum states obtained by solving the Schrödinger equation for the wavefunction of the nanosphere in this potential.

The commonly used Casimir-Polder formula, which also corresponds to the limit of our calculations for a vanishingly small radius $R$, leads to significant difficulties since it predicts a power law $|E| \propto R^{3} / L^{3}$ in the vicinity of the surface and thus leads to a ill-behaved Schrödinger problem. The exact solution presented in the present paper for a finite value of the radius $R$ predicts a smoother power law $|E| \propto R / L$ in the vicinity of 
the surface and thus leads to a regular solution for the Schrödinger equation [9].

The plate roughness has been disregarded here and it is treated in a phenomenological manner in 9]. It would be interesting to analyze its effect by using techniques already developed for treating the scattering on rough surfaces 31 33].

It would also be worth investigating the same problem for the interaction between an atom and a plane. In analogy with the discussion of the present paper, taking into account the higher order multipoles and multiple interferences could lead to an expression of the energy more regular than with the commonly used electric dipole approximation

\section{Acknowledgments}

The authors thank M.-C. Angonin, I. Cavero-Pelaez, G.-L. Ingold, R. Messina, H.M. Nussenzveig, S. Pelisson and P. Wolf for discussions, and the ESF Research Networking Programme CASIMIR (www.casimirnetwork.com) for providing excellent opportunities for discussions on the Casimir effect and related topics. PAMN thanks CNPq and FAPERJ for financial support.
[1] H.B.G. Casimir, Proc. K. Ned. Akad. Wet. 51793 (1948)

[2] P.W. Milonni, The Quantum Vacuum (Academic Press, 1994)

[3] S.K. Lamoreaux, Am. J. Phys. 67850 (1999)

[4] A. Lambrecht, A. Canaguier-Durand, R. Guérout, S. Reynaud, in Casimir Physics (Lecture Notes in Physics, Springer, 2011) eds D. Dalvit, P. Milonni, D. Roberts and F. da Rosa arXiv:1006.2959

[5] V.A. Parsegian, Van der Waals Forces: a Handbook for Biologists, Chemists, Engineers, and Physicists (Cambridge University Press, 2006)

[6] V.V. Nesvizhevsky, Phys. Atomic Nuclei 65400 (2002)

[7] E.V. Lychagin et al, Phys. Atomic Nuclei 651995 (2002)

[8] D.G. Kartashov et al, Int. J. Nanoscience 6501 (2007)

[9] V.V. Nesvizhevsky, A.Yu. Voronin, A. Lambrecht, S. Reynaud et al, in preparation (2011)

[10] H.B.G. Casimir and D. Polder, Phys. Rev. 73360 (1948)

[11] A. Lambrecht, P.A. Maia Neto and S. Reynaud, New J. Phys. 8243 (2006)

[12] I.E. Dzyaloshinskii , E.M. Lifshitz and L.P. Pitaevskii, Sov. Phys. Uspekhi 4153 (1961)

[13] P.A. Maia Neto, A. Lambrecht and S. Reynaud, Phys. Rev. A78 012115 (2008)

[14] A. Canaguier-Durand, P.A. Maia Neto, I. Cavero-Pelaez, A. Lambrecht and S. Reynaud, Phys. Rev. Lett. 102 230404 (2009)

[15] A. Canaguier-Durand, P.A. Maia Neto, A. Lambrecht and S. Reynaud, Phys. Rev. Lett. 104040403 (2010)

[16] A. Canaguier-Durand, P.A. Maia Neto, A. Lambrecht and S. Reynaud, Phys. Rev. A82 012511 (2010)

[17] T. Emig, J. Stat. Mech. P04007 (2008)

[18] T. Emig, Int. J. Mod. Phys. A25 2177 (2010)
[19] G.L. Ingold, A. Lambrecht and S. Reynaud, Phys. Rev. E80 041113 (2009)

[20] H.M. Nussenzveig Diffraction Effects in Semiclassical Scattering (Cambridge: University Press Cambridge, 1992)

[21] P. Johannson and P. Apell, Phys Rev. B56 4159 (1997)

[22] C. Noguez, C.E. Roman-Velazquez, R. Esquivel-Sirvent and C. Villarreal, Europhys. Lett. 67191 (2004)

[23] G. Celep, E. Cottancin, J. Lermé et al, Phys Rev. B70 165409 (2004)

[24] E. Cottancin, G. Celep, J. Lermé et al, Theor. Chem. Acta 116514 (2006)

[25] C.F. Bohren and D.R. Huffman, Absorption and Scattering of Light by Small Particles (Wiley, New York, 1983), ch. 4.

[26] D.A.R. Dalvit, P.A. Maia Neto, A. Lambrecht, and S. Reynaud, Phys. Rev. Lett. 100040405 (2008)

[27] R. Messina, D.A.R. Dalvit, P.A. Maia Neto, A. Lambrecht and S. Reynaud, Phys. Rev. A80 022119 (2009)

[28] H.C. Hamaker, Physica 41058 (1937)

[29] B.V. Derjaguin and I.I. Abrikosova, Sov. Phys. JETP 3 819 (1957)

[30] C. Genet, A. Lambrecht and S. Reynaud, Ann. Fond. L. de Broglie 29311 (2004)

[31] P.A. Maia Neto, A. Lambrecht and S. Reynaud Europhys Lett. 69924 (2005)

[32] P.A. Maia Neto, A. Lambrecht and S. Reynaud Phys Rev. A72 012115 (2005)

[33] A.M. Contreras-Reyes, R. Guérout, P.A. Maia Neto, D.A.R. Dalvit, A. Lambrecht and S. Reynaud Phys Rev. A82 052517 (2010) 